\title{
An advanced management architecture for IN
}

\author{
Dominique Gaïti \\ Columbia University \\ Center for Telecommunications Research, \\ Room 801, Schapiro Research Building, \\ New York, NY 10027-6699, USA \\ Tel: (212) 854 8928, Fax: (212) 3169068 ,
}

Email: gaiti@ctr.columbia.edu

\begin{abstract}
To provide various advanced telecommunications services and to enhance their operations, it is necessary to introduce a flexible and adaptable architecture on the Intelligent Network (IN). IN may be seen as one of the most innovative concepts of that last decade, if this flexibility and adaptability may be achieved. One way to reach such a goal is to introduce some intelligence. In that way, we propose a new management architecture for IN introducing concepts from the field of Distributed Artificial Intelligence.

First, this paper describes a proposed generic architecture to support the IN management aspects. Then, to provide an intelligent architecture, we investigate the field of Distributed Artificial Intelligence, and a model adapted to these new concepts is proposed. Finally, we propose the integration of the generic IN management architecture and the intelligent model to provide an intelligent architecture for IN management.
\end{abstract}

\section{INTRODUCTION}

The objective of Intelligent Networks (IN) is to allow the introduction of new capabilities in telecommunication networks and to facilitate and accelerate, in a cost-effective manner, service implementation and provisioning in a multivendor environment. IN is a generic serviceoriented architecture intended to be used for all kinds of services. The management aspects of IN are mainly focused on "service management", that is, management related to services on an IN architecture.

A conceptual model for IN was developed under the auspices of ITU-T (ex CCITT) to aid in the specification of IN. Functional and physical entities have been defined in this model. In 
particular, some functions are devoted to network management of IN services. A network management architecture is necessary to realize the functions described in these management entities. In this paper we provide an advanced management architecture well adapted to these IN concepts. This architecture uses some ideas developed in the Distributed Artificial Intelligence field.

The paper is in four sections. The first section describes IN concepts and the work focusing on IN management aspects. Section 2 proposes a generic architecture to support these Intelligent Network management aspects. To provide an intelligent management architecture, we investigate the field of Distributed Artificial Intelligence described in section 3. In section 4 the integration of sections 1 through 3 is realized to provide an intelligent architecture for $\mathbb{N}$ management.

\section{INTELLIGENT NETWORK MANAGEMENT ASPECTS}

The IN Conceptual Model (INCM) was defined by ITU-T as a methodology for specifying the IN architecture. It is not an architecture in itself, but a framework for the design and description of the IN [1] [2]. The INCM consists of four planes (Figure 1), where each plane represents a different abstract view of the capabilities provided by an IN-structured network. The planes represent the service view, the global functional view, the distributed functional view, and the physical view of $\mathbb{I N}$.

The service plane gives an abstract end-user view of the services provided by an INstructured network. A service can be decomposed into one or more service features. A service feature is built from several Service Independent Building blocks.

The global functional plane defines the Service Independent Building blocks (SIBs) with the following principle: the network is viewed as a single virtual machine. It turns out that the distribution is transparent at that level.

The distributed functional plane models a distributed view of an Intelligent Network. This view consists of Functional Entities (FEs). A FE is a unique group of functions in a single location and a subset of the total set of functions required to provide the service feature supported by the SIB. A group of centralized or distributed FEs is mapped onto a SIB. Then a SIB is realized by the interaction between corresponding distributed FEs. The advantage of this representation is to have reusable FEs, i.e., a FE can be used by several SIBs.

Three types of management-related functional entities are provided: Service Management Function (SMF), Service Management Access Function (SMAF), and Service Creation Environment Function (SCEF).

- A Service Management Functions (SMF) supports deployment and provision of IN provided services and/or ongoing operations. Particularly, for a given service, it supports co-ordination of different Service Control Function (SCF) and Service Data Function (SDF) instances, e.g.:

- billing and statistical information are received from the SCFs, and made available to authorized service managers through the SMAF;

- modifications in service data are distributed in SDFs that keep track of the reference service data values. 
The SMF manages and updates service related information.

- A Service Management Access Function (SMAF) provides an interface between the service managers and the Service Management Function. This service includes checking authority to access function.

- A Service Creation Environment Function (SCEF) allows services provided in Intelligent Network to be defined, developed, tested and made supportable by Service Management Functions. Output of this function includes service data definitions and service trigger definitions.

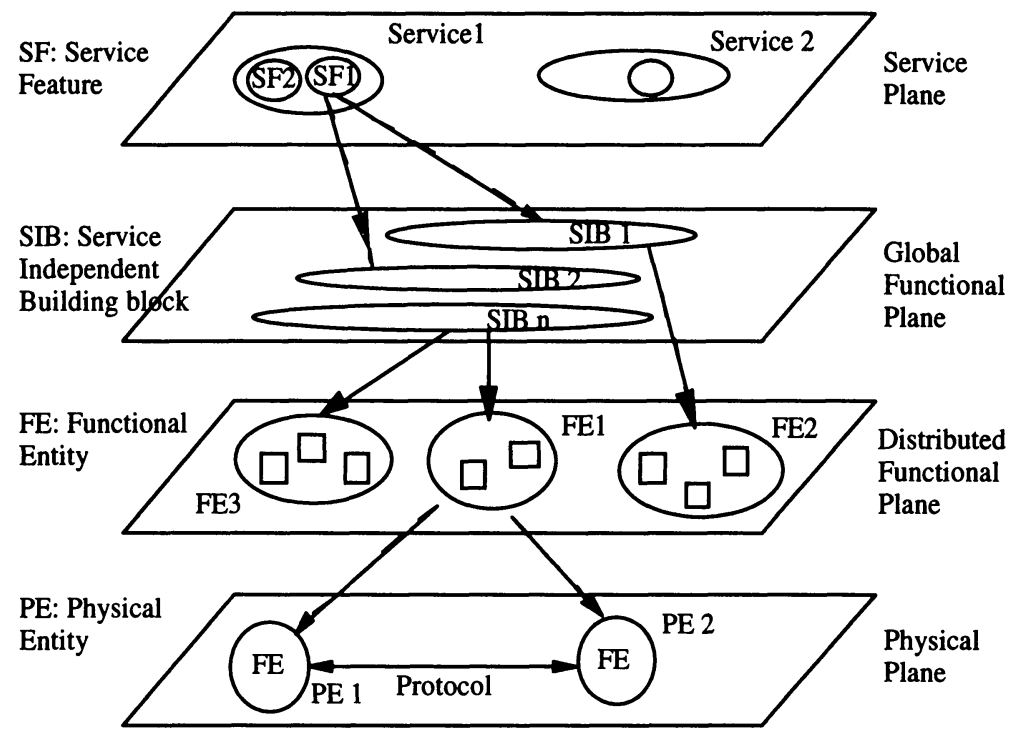

Figure 1. The Intelligent Network Conceptual Model.

The physical plane identifies the different Physical Entities (PEs). A Physical Entity consists of one or more Functional Entities. One or more FEs may be mapped onto the same PE, but a single FE cannot be split between two PEs [3]. This plane takes into account physical considerations, optimization aspects, and protocols.

In this paper, we are mainly interested in Service Management Functions (SMFs). They handle five activities (service deployment, service provisioning, service control, billing, and service monitoring). The SMFs are located in the Service Management Points (SMPs). Now the problem is how to design these SMFs to co-operate in an intelligent way? We have to define what resources are managed by what SMPs. How are the SMAFs realized given, that they are located in an intelligent terminal or a computer? What is the structure of an SMP?

In the next section we propose an architecture able to support IN management and to answer the different problems arising in this area. 


\section{MANAGEMENT ARCHITECTURE}

We are going to define an architecture for integrating the management of all the resources of a distributed system. The architecture we have adopted has been defined in a previous paper [4] and is derived from proposals developed in the European Esprit or Race projects [5] [6] [7] [8] [9]. We defined a generic integrated management architecture through an object-oriented approach able to model all entities of a distributed management systems. This allows us to represent and to manipulate in a uniform way the information coming both from the attributes of the managed entities, and from management entities. An object machine permits basic services like creation and removing of types of objects and instances. This object machine should also support location-independent interactions between objects (invocation) and protection against wrong object use.

Management is modelled in the following terms:

- The resources whose activity produces a specific function for the treatment of the information;

- A manager that provides the three following functions:

- A monitoring function to supervise resources and activities to insure that they are conform to a management policy, and to identify the possible conflicts;

- A control function to select a control operation following an algorithm determined by the policy management;

- A default function to describe what to do when the management of resources and activities cannot be fulfilled following the defined policy.

Let us note that a manager may be responsible for several resources, each of them subject to control and supervision.

The management is hierarchical to facilitate flexible implementation and future extensions. A manager may itself be managed by a higher level manager. It turns out that a manager can work with a certain autonomy depending on its own policy. At the same time, a manager may answer when it receives a control command. The basic model can be extended recursively to different levels of refinement and specialization.

The management is also multiple. It is necessary to share the resources that are managed simultaneously by several managers. It is also necessary to establish a negotiation between the managers to avoid conflicts due to concurrent control of the same resource.

Our management architecture defines the basic principles of organization of the IN world and the structures that describe it.

The domain is a first principle for the organization. A domain (from a management point of view) consists of resources to be managed in a distributed system and a specific management policy (or a component of this policy).

The second organizing principle concerns the Management Process (MP), which includes the hardware, the software and, the people executing the process. For example, we can group the objects to be managed according to the level where they are or the relationship between the different layers. The Management Process contains two components: 
- The MP view, which is the abstract representation of the resources and the activities under control. It is possible to have a set of views that corresponds to different subsets of resources.

- The MP core that executes the management process. It manages and activates the resources through the MP view.

This distinction between the MP view and the MP core allows the separation of the management policy (namely, the goal of the management system) from the resources and the managed activities (namely, the managed objects). These principles are shown in Figure 2.

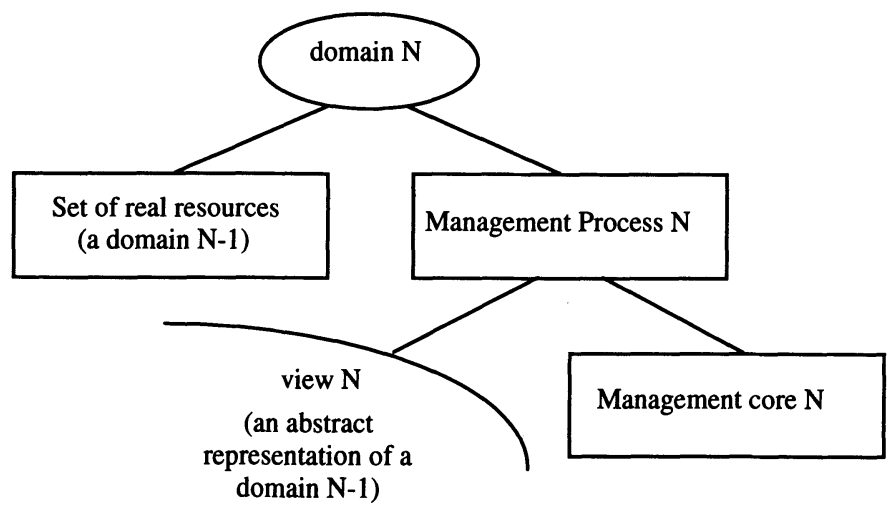

Figure 2. The principles of structure.

The concept of domain allows the description of a large number of management cases, from simple managed resources to very complex structures. Indeed, the domains may be structured to reflect a delegate administration in relation with another domain. This delegation may be applied in a recursive way. It means that a domain $\mathrm{N}$ may delegate to another domain $\mathrm{N}-1$ the management of some of its real resources. The abstract representation of $\mathrm{N}-1$ constitutes the part of the MP view of N. It turns out that N-1 represents a subset of N's resources. This decomposition may facilitate the management application.

This domain and MP-based architecture allows the domains to be completely autonomous since they are able to manage their own resources. This permits the description of a managed resource to be provided very easily. Moreover, the delegation of some management tasks by one domain to another allows the creation of complex schemes. Such an approach allows the modelling of layered structures with their dependencies.

The use of Knowledge Based Systems (KBS) lets one capture network management knowledge. One of the main features of a network management system is the large amount of information it handles. Looking at this knowledge as one big area would make it impossible to find a single representation formalism covering everything. But we can adopt a top-down approach and try to find some of the main objects in this large area.

We have found three objects on a very high level that we think cover a large part of our management system and can be fairly representative. These objects are the network, the 
services, and the users of the management system. For each object, we have proposed knowledge representation formalisms capturing the knowledge properly [10].

\section{TOWARD AN INTELLIGENT MANGEMENT ARCHITECTURE}

\subsection{Distributed Artificial Intelligence Concepts}

Real time and distribution problems are two fundamental aspects of network management. In fact, time constraints are crucial for telecommunication system features such as response time (from user point of view) and performance throughput (from the network-manager point of view). Network applications require very short response times when performing for example, routing table up-date or repairs in case of various network faults (e.g., network overload, hardware failure).

The aim is to keep the user unaware of problems occurring in the network. Consequently, the network manager should be able to detect immediately any network malfunction (fault detection), distinguish its real causes from the numerous side effects (fault isolation), and identify the problem (diagnosis). Then, he should ask for the required knowledge for building a solution to restore the network to an unfaulty behavior (effective repair). So, the required expert system has a difficult task to encompass all aspects of the above operations.

Moreover, a network is characterized by its heterogeneity, its layered structure, and the distribution of its resources. Thus, we have to formalize such a network by a system in which knowledge is distributed [11].

Distributed Artificial Intelligence (DAI) allows several processes, called agents, to solve a single problem. Each process uses local power and communicates with remote hosts. In the DAI field, the research aim is to propose a scheme of organization allowing the distribution of the resolution of a shared problem. This organization has to describe the mechanisms that can be used by agents to co-operate.

With DAI, problem solving involves the co-operation of decentralized processes. Agents may be small real processes or they can be complex real ones such as applications based upon large knowledge bases. The problem solving process is distributed, meaning that each agent has to share a common information set allowing the entire group to reach a solution. Decentralized processing means that data and control are often logically and physically distributed [12].

\subsection{Distributed Artificial Intelligence Architecture}

The implementation of a DAI architecture may follow different techniques [13]. In our project, the DAI architecture follows the principle of the blackboard architecture [14]. It incorporates three components (Figure 3) [15]:

- A structured knowledge base called "blackboard" that contains the current state of the solution for a given problem. This shared data area is analogous to the working memory accessed by many production rules in a rule-based system. It is divided into separate areas of semantic abstraction called levels. 
- A collection of independent agents (or knowledge sources) which may read and write one or several levels. They can be seen as a collection of independent processes able to cooperate to solve a problem. Each agent is specialized in one particular sub-domain.

- A control system that insures the supervision of the actions of the different knowledge sources. The control system is also a collection of integrated knowledge sources.

This organization is not sufficient to realize a real multi-agent system. We need to develop a real architecture for the agent itself; it cannot be just a collection of rules, an entire procedure, or a whole rule-based system.

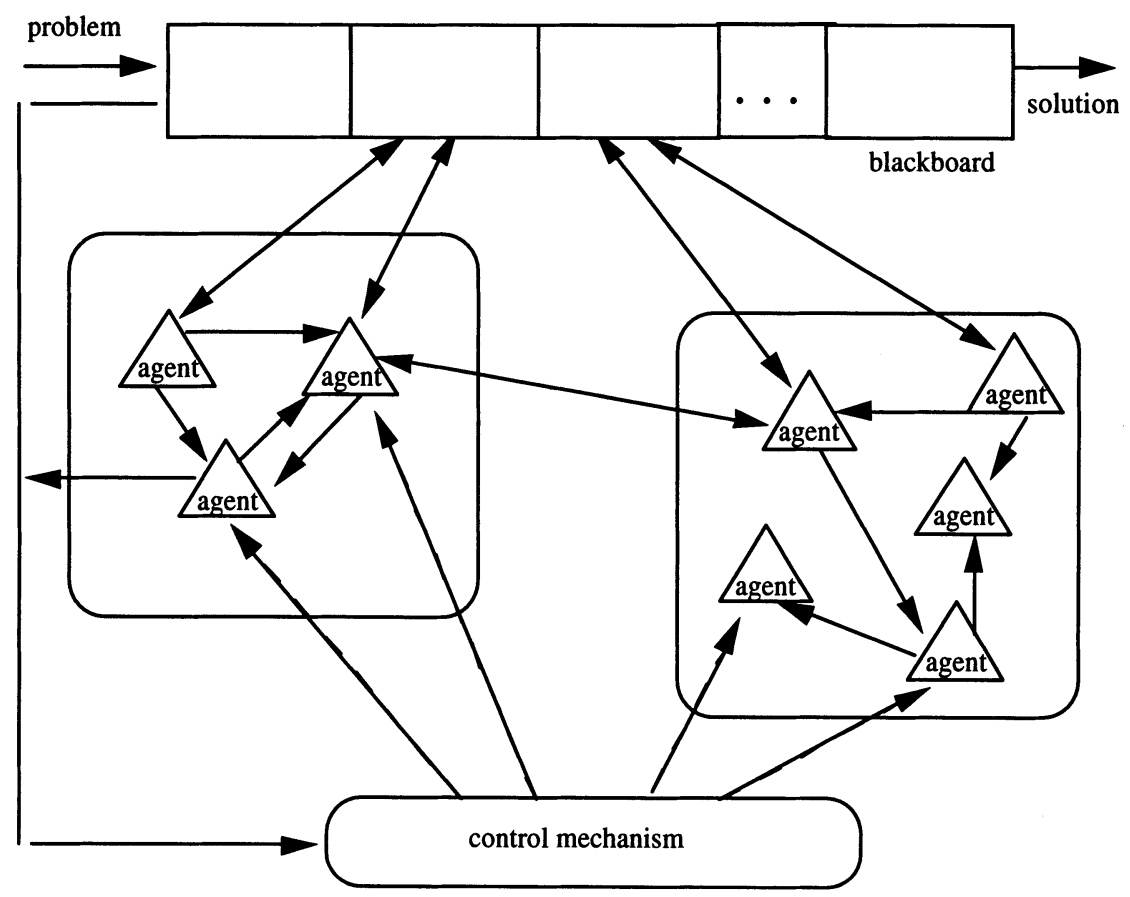

Figure 3. The blackboard architecture.

The agent itself is organized following the principles of the blackboard architecture. It has three components (Figure 4):

- a knowledge module: this relates to all the usual data and the knowledge of the domain;

- a control module: this defines the problem-solving strategy and the control mechanism to determine the next action to perform. It corresponds to the inference engine in the rulebased system;

- a communication module: this gives a model of the other agents in the environment. It also provides an interpreter of messages and a logic for communications with the other agents.

Each agent may be seen as an independent software processor, with its own resources. The agents communicate by sharing the information if we look at a high level, i.e., at the entire 
multi-agent system level. They communicate through the blackboard that contains, at the beginning, the facts of a given problem. The agents read the information in the blackboard and write in it to reach a solution step by step.

If we look at a lower level, the communication module in each agent is in charge of receiving and sending messages to the other agents to co-operate in building a solution if they do not have enough knowledge to solve the problem themselves.

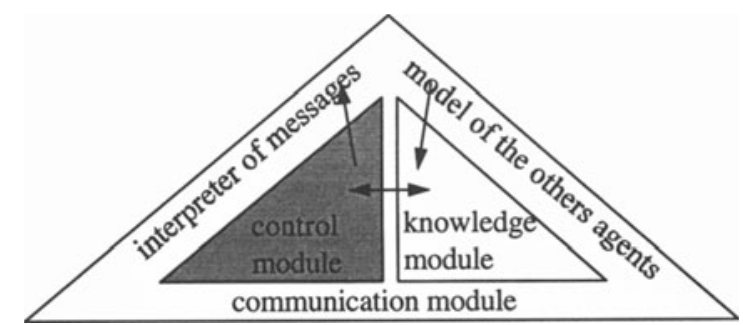

Figure 4. An agent.

\subsection{An Intelligent Integrated Architecture}

In $\S 1$ and $\S 2$, we defined a generic integrated management architecture. Now we want to apply the DAI model on this architecture to get an Intelligent Integrated Architecture.

First, we have to define the different domains. The domains are composed of a set of managed resources but also encapsulate the components performing management. Therefore, management components and resources may be grouped to build domains according to different criteria associated with the contained management resources (type, location, functionality, ownership, or objectives (management function, applied policy, authority)). For example, if we are interested in the management of $\mathrm{N}$ interconnected sub-networks, a domain can be a sub-network. But, a domain may be constituted with the machines of a manufacturer $\mathrm{X}$ or may be constituted with the software and hardware of the layer $\mathbf{n}$ of the OSI architecture (on all the network or on a sub-network or only on a part of the network).

The domain is managed by one or several agents that can be situated in any node of the network belonging to the domain. These agents work in a co-ordinated manner on a common data structure: the blackboard. This is situated in the MP core where all the management functions are provided. The agents may work in group on a given problem (in the blackboard) or may work alone or with some other agents of their environment to solve more trivial problems when they have the appropriate knowledge. The structure of the agents and the way they are organized are described in $§ 3.2$.

\section{AN ADVANCED MANAGEMENT ARCHITECTURE FOR IN}

In the IN, several scenarios for the physical architecture can be deployed. We propose to adapt our intelligent management architecture on the physical plane, namely the last plane of Figure 
1. This plane is decomposed into domains. They are defined following different criteria, depending on the structure of the network, the needs of the operator, the user constraints, and the diversity of the infrastructure. An example of a domain is illustrated in Figure 5.

The domain is composed of one Service Management Point (SMP) that performs service management control, service provision control, service deployment control, etc. Examples of functions that the SMP can perform are database administration, network surveillance and testing, network traffic management, network data collection. Functionally, the SMP contains the Service Management Function (SMF) and, optionally, the Service Management Access Function (SMAF) and the Service Creation Environment Function (SCEF). The SMP can access all other PEs.

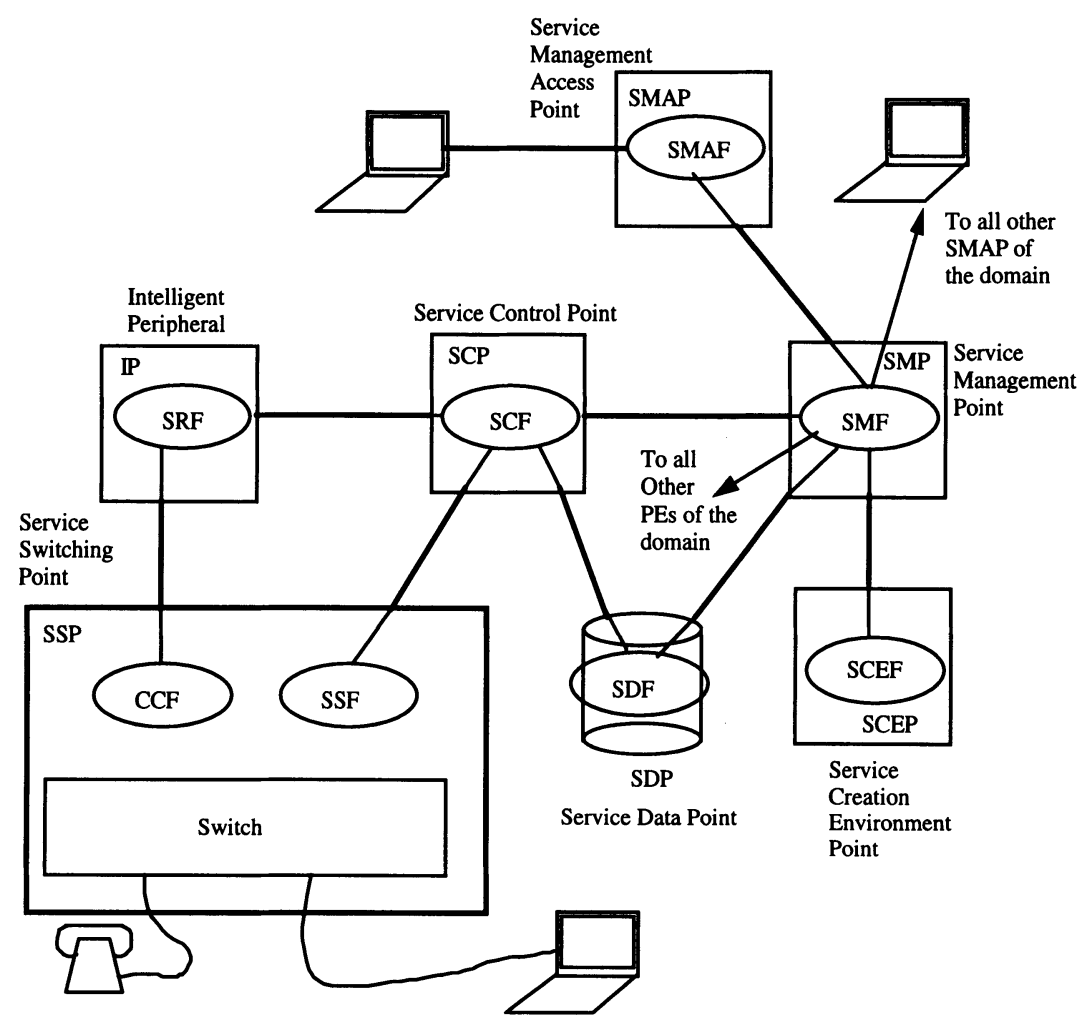

Figure 5. A domain.

In Figure 5, the SMAF and SCEF functions are performed in different Physical Elements. The SMAF is included in a Service Management Access Point (SMAP). This SMAP may be used as a service independent front end to the SMP or to all SMPs of the system. This simplifies the user's procedures (customer or network operator) as there would be only one entry point for the management of all IN-structured services. In addition, all security-related data for user identification, for example, may be specified for each SMAF. These functions 
need not be grouped together as it happens when the SMAF is located in the SMP. The SCEF is included in the Service Creation Environment Point (SCEP) that is used to define, develop and test an IN service, and to input it into the SMP.

Other Physical Entities can be Service Switching Point (SSP), Service Control Point (SCP), Service Data Point (SDP), Intelligent Peripheral (IP), Service Switching and Control Point (SSCP), Service Node (SN) and so on.

For each domain a Service Management Point (SMP) is defined. The MP core is situated in this SMP and is in charge of the Service Management Functions. At each IN Functional Entity there is an agent that can communicate with other agents and with the blackboard situated in the MP core. The management relationship between the SMF and any FEs is performed through the communication between the agents and the blackboard. The information flows between the SMF and the other functional entities are defined by the agent capabilities. Moreover, a pool of agents is provided in the Service Creation Environment Point (SCEP) supporting the Service Creation Environment Function (SCEF). For example, the SCEP may contain an agent for service specification, an agent for service analysis, an agent for service generation, and an agent for service testing.

The architecture we described in Figure 5 describes one domain. Now, a general topology of our global architecture is illustrated in Figure 6.

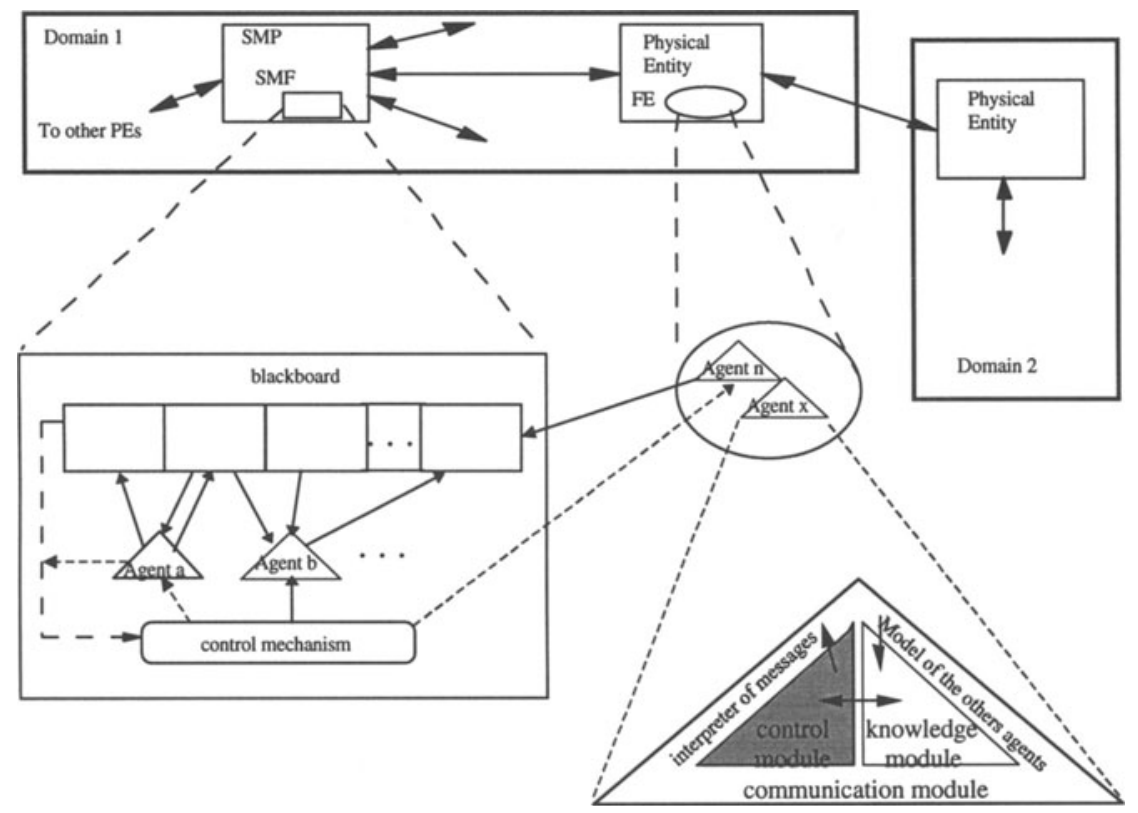

Figure 6. An intelligent management architecture for IN. 
In this figure two domains are shown. Each domain contains one Service Management Point with one blackboard and a set of agents attached to this blackboard.

The blackboards and the agents of the different domains may co-operate to insure the global management of the system. The main problem is to organize the global system to define a general service management process able to take charge of a global service. This can be performed through a hierarchical structure of distributed systems.

We may define a main agent, situated in a central control node. This main agent has the same structure we described before with three components but not the same functions:

- A knowledge module containing the awareness of all the other agents in the global system. It means that the main agent knows exactly which agent is able to solve a particular task. It also has knowledge concerning the way to subdivide a global problem into sub-problems and the way to put together partial results to obtain a global solution for a global problem;

- A control module: it fulfills the same functions as in ordinary agents;

- A communication module: it gives a model of the different blackboards in the environment. It also provides a way to read and write in these blackboards.

When a general problem appears, the main agent subdivides it into sub-problems and looks for the agents able to solve a particular sub-problem. When it finds them, it writes sub-problem data into their associated blackboards. It can write in different blackboards.

The main agent reads from these blackboards after the agents finish their work and puts together all the partial results it found to achieve a global solution.

\section{CONCLUSIONS}

This paper proposes a framework for defining an intelligent and integrated model to be applied in the service management area of the Intelligent Network. The main idea was to reduce the complexity of the management process. We dealt with complexity by introducing agents and blackboards as intelligent concepts. In this paper, we worked on a low time scale corresponding to service management tasks. In a future issue, we would like to enlarge the applicability of the architecture by introducing new concepts in the agents to take care of real time considerations.

\section{REFERENCES}

[1] ITU-T I.312/Q.1201 Principles of Intelligent Network Architecture, October 1992, Geneva.

[2] José M. Duran and John Visser, International Standards for Intelligent Networks, IEEE Communications Magazine, February 1992

[3] W. Ambrosch, A. Malher, B. Sasscer, The Intelligent Network, Springer-Verlag, 89.

[4] D. Gaiti, I2NMA: an Intelligent Integrated Network Management Architecture, International Journal on Network Management, vol 4, 3, 1994.

[5] The final technical Report of RACE project ADVANCE (R1009), 1992. 
[6] The final technical Report of RACE project NEMESYS (R1005), 1992.

[7] The final technical Report of RACE project AIM, (R1005), 1992.

[8] ROSA Architecture - Final release, RACE project 1093, April 1992.

[9] M. Möller et al., DOMAINS Basic Concepts for Distributed Systems Management, Proceedings of the fifth RACE TMN Conference, 1991.

[10] I. Rahali and D. Gaiti, A Multi-agent System for Network Management, In Integrated Network Management II, pp. 469-479, Ed. I. Krishnan \& W. Zimmer, Elsevier Science Publishers B.V. (North Holland), 1991.

[11] D. Gaiti and I. Rahali, Applying Artificial Intelligence Techniques to the Management of Heterogeneous Networks, In Network Management and Control, pp. 189-200, Ed.

A. Kershenbaum, M. Malek, M. Wall, Plenum Publishing corporation, 1990.

[12] L. Gasser, Distributed Artificial Intelligence, AI Expert, July 89.

[13] E. Werner, The Design of Multi-Agent Systems, Decentralized A.I.-3, Elsevier Science Publishers B.V., 1992.

[14] R. Engelmore et al., Blackboard Systems, Reading, Mass.: Addison-Wesley, 1988.

[15] D. Gaiti, Distributed Problem Solving in Network Management, Proc. of International Conference IFIP/IEEE on Distributed System: Operation and Management, Long Branch N.J., USA, October 1993. 